\title{
Paediatric dental sedation: Will your child return home unharmed?
}

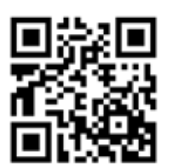

Sedation is widely used to reduce the fear and anxiety associated with dental procedures. In South Africa (SA), this is frequently done in consultation rooms rather than a theatre environment, with the aim of reducing cost and improving patient comfort, particularly in the case of children. Both local and systemic analgesia are combined with sedation. While severe complications are rare, critical incidents such as dysrhythmias or respiratory depression are common. Adverse events occur more frequently and have worse outcomes in the younger age group ( $<7$ years of age) ${ }^{[1]}$ Such events include undersedation (with movement and possible injury), oversedation, oxygen desaturation, airway obstruction, laryngospasm, respiratory and cardiovascular depression or respiratory or cardiac arrest, seizures, unresponsiveness, allergic reactions, vomiting or excessive secretions, with risk of aspiration, and death.

Sedation is by nature a continuum from minimally reduced responsiveness to general anaesthesia. Children can easily slip into a deeper level of sedation with respiratory or cardiovascular compromise, depending on individual susceptibility. This tends to happen more readily when more than one drug is used. For this reason, close observation and recorded monitoring, as well as appropriate training in sedation, are essential. Lack of well-kept records is one reason why complications of sedation of children in the dental chair are underestimated. The South African Society of Anaesthesiologists compiled paediatric sedation guidelines in 2010, ${ }^{[2]}$ due for update later in 2015. According to this guideline (and other international guidelines), whenever sedation is attempted in children, personnel must be appropriately trained and include an observer who is not the surgeon. There must be monitoring with appropriate equipment, and resuscitation equipment and resuscitation and antidote drugs must be available.

This issue of $S A M J$ features a well-planned and well-conducted audit of sedation practices in Gauteng Province, SA. ${ }^{[3]}$ The findings are worrying. In only $76 \%$ of practices was informed consent obtained before procedures, while only $83 \%$ of patients had presedation assessments. Although 41\% (95\% confidence interval 37 - 51) of dentists provided sedation, only $78 \%$ had oxygen available. Almost $20 \%$ had no resuscitation equipment available in their practice. Facial masks and airway equipment were present in only $30 \%$ of practices. During sedation only $54 \%$ of respondents used pulse oximetry, the single means of monitoring that is considered essential for any level of sedation; more than $41 \%$ had no monitor available. In $41 \%$ of practices the dentist provided the sedation, did the procedure and monitored the child's condition. Two-thirds of practitioners used more than one drug, yet half kept no emergency drugs: flumazenil, an antidote to benzodiazepines, was kept by only 10 $15 \%$ of practitioners who used benzodiazepines, and only $14 \%$ of practitioners using opiates stocked the antidote naloxone. A positive aspect is that in $31 \%$ of practices a medical practitioner with training in sedation, and in almost $21 \%$ of practices an anaesthetist, was the person primarily responsible for sedation. However, that leaves $48 \%$ of children sedated by someone with no training in sedation. The majority (90\%) of personnel primarily responsible for sedation had Basic Life Support training.

The study ${ }^{[3]}$ may be criticised, as numbers were relatively small, and despite good sample size planning a relatively low proportion of the dentists completed the questionnaire. However, although the final number of respondents was only 52, the results probably represent fairly accurately what currently happens in practice. It is also possible that people who did not respond did not want to provide information on practices that they do not feel comfortable with.

It is clear that many children in the dental chair may be at risk - in a situation where complications may not be detected early, and where there is a lack of appropriate knowledge and of drugs or equipment to reverse inappropriately deep sedation and ensure resuscitation. Poor appreciation of the risks and of guidelines, and inadequate training, further aggravate the situation. Fortunately, $82 \%$ of respondents stated that they wanted to attend a sedation course.

Dental chair sedation is also influenced by cost considerations. According to guidelines, deeper levels of sedation should only take place in a controlled theatre/hospital situation, but medical insurers resist such practice for relatively minor procedures. Medical funders also sometimes refuse to pay for a dedicated sedationist, such as an anaesthetist. If sedation is done in the office, it is the responsibility of the dental practitioner to stock all appropriate drugs and monitoring and resuscitation equipment, all of which is also a cost factor.

What should be done? In a developing country such as SA, which is able to provide First-World medicine, the current situation is unacceptable, whether in the best- or the least-funded situation. Acknowledging that even the mildest sedation for an apparently minor procedure may go wrong, the status quo must be improved so that complications, should they arise, will be successfully managed with a good outcome. Awareness of problems that may be associated with dental chair sedation is essential. Training in sedation is vital. Practice should be according to national and international guidelines, with awareness of and ability to determine the level of sedation. No sedation should be attempted without ready availability of essential drugs and monitoring and resuscitation equipment.

Ultimately a system of accreditation of training and facilities should be created, supervised and inspected by the regulating authority.

\section{B J S Diedericks}

Department of Anaesthesiology, Faculty of Health Sciences, University of the Free State, Bloemfontein, South Africa

\section{Corresponding author: B J S Diedericks (diedericksbjs@ufs.ac.za)}

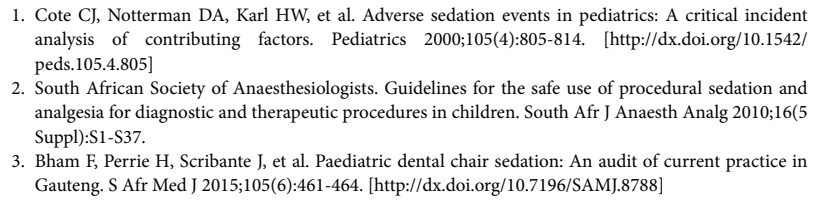
analysis of contributing factors. Pediatrics 2000;105(4):805-814. [http://dx.doi.org/10.1542 peds.105.4.805]

2. South African Society of Anaesthesiologists. Guidelines for the safe use of procedural sedation and analgesia for diagnostic and therapeutic procedures in children. South Afr J Anaesth Analg 2010;16(5 Suppl):S1-S37.

3. Bham F, Perrie H, Scribante J, et al. Paediatric dental chair sedation: An audit of current practice in Gauteng. S Afr Med J 2015;105(6):461-464. [http://dx.doi.org/10.7196/SAMJ.8788]

S Afr Med J 2015;105(6):453. DOI:10.7196/SAMJ.9756 